\title{
QTL mapping of web blotch resistance in peanut by high-throughput genome-wide sequencing
}

\author{
Hua Liu ${ }^{1,2+}$, Ziqi Sun ${ }^{2+}$, Xinyou Zhang ${ }^{2 *}$, Li Qin $^{2}$, Feiyan Qi ${ }^{2}$, Zhenyu Wang ${ }^{3}$, Pei Du ${ }^{2}$, Jing Xu², Zhongxin Zhang ${ }^{2}$, \\ Suoyi Han², Shaojian Li $i^{3}$, Meng Gao ${ }^{3}$, Lina Zhang ${ }^{2}$, Yujie Cheng ${ }^{2}$, Zheng Zheng ${ }^{2}$, Bingyan Huang ${ }^{2}$ and \\ Wenzhao Dong ${ }^{2}$
}

\begin{abstract}
Background: Web blotch is one of the most important foliar diseases worldwide in peanut (Arachis hypogaea L.). The identification of quantitative trait loci (QTLs) for peanut web blotch resistance represents the basis for gene mining and the application of molecular breeding technologies.

Results: In this study, a peanut recombinant inbred line (RIL) population was used to map QTLs for web blotch resistance based on high-throughput genome-wide sequencing. Frequency distributions of disease grade and disease index in five environments indicated wide phenotypic variations in response to web blotch among RILs. A high-density genetic map was constructed, containing 3634 bin markers distributed on 20 peanut linkage groups (LGs) with an average genetic distance of $0.5 \mathrm{cM}$. In total, eight QTLs were detected for peanut web blotch resistance in at least two environments, explaining from 2.8 to $15.1 \%$ of phenotypic variance. Two major QTLs qWBRA04 and qWBRA14 were detected in all five environments and were linked to 40 candidate genes encoding nucleotide-binding site leucine-rich repeat (NBS-LRR) or other proteins related to disease resistances.
\end{abstract}

Conclusions: The results of this study provide a basis for breeding peanut cultivars with web blotch resistance.

Keywords: QTL mapping, Peanut, Web blotch resistance, Resequencing

\section{Background}

Cultivated peanut (Arachis hypogaea L.) is one of the most important oil legumes in many countries. A. hypogaea is an allotetraploid (AABB, $2 n=4 x=40$ ) with the genome size of $\sim 2.7 \mathrm{~Gb}$ [1]. The genome assembly results of two prominent parental cultivars of many Chinese peanut varieties "Fuhuasheng" and "Shitouqi" and one American cultivar "Tifrunner" were published in 2019

\footnotetext{
*Correspondence: haasz@126.com

${ }^{+}$Hua Liu, Ziqi Sun and Xinyou Zhang contributed equally to this work.

${ }^{2}$ Industrial Crops Research Institute, Henan Academy of Agricultural Sciences / Key Laboratory of Oil Crops in Huang-Huai-Hai Plains, Ministry of

Agriculture and Rural Affairs / Henan Provincial Key Laboratory for Genetic Improvement of Oil Crops, Zhengzhou 450002, PR China

Full list of author information is available at the end of the article
}

[2-4]. The assembled sequences were $\sim 2.54 \mathrm{~Gb}$ and the predicated genes were about $60 \sim 80$ million [2-4].

Peanut web blotch, also called muddy spot or net blotch [5, 6], caused by Phoma araehidieola Marasas, Pauer \& Boerema $[7,8]$, is one of the most important foliar diseases in peanut. Peanut web blotch was first reported in Texas (U.S) in 1970s [9] and then, in the early 1980s, it was found in the major peanut growing areas of Shandong and Liaoning provinces of China [10, 11]. Subsequently, it was discovered in Shanxi province [12] and Henan province [13]. The web blotch disease can occur during the whole peanut growing period and can cause yield losses of $10 \% \sim 20 \%$ usually, but with the heaviest of more than $50 \%$. Therefore, it has been

(c) The Author(s). 2020 Open Access This article is licensed under a Creative Commons Attribution 4.0 International License, which permits use, sharing, adaptation, distribution and reproduction in any medium or format, as long as you give appropriate credit to the original author(s) and the source, provide a link to the Creative Commons licence, and indicate if changes were made. The images or other third party material in this article are included in the article's Creative Commons licence, unless indicated otherwise in a credit line to the material. If material is not included in the article's Creative Commons licence and your intended use is not permitted by statutory regulation or exceeds the permitted use, you will need to obtain permission directly from the copyright holder. To view a copy of this licence, visit http://creativecommons.org/licenses/by/4.0/ The Creative Commons Public Domain Dedication waiver (http://creativecommons.org/publicdomain/zero/1.0/) applies to the data made available in this article, unless otherwise stated in a credit line to the data. 
regarded as one of the most urgent issues to be addressed in some peanut growing areas.

The fungus $P$. araehidieola pathogens normally overwinter within the crop residue and plants of all ages are susceptible [6]. Cloudy weather with frequent rains and temperatures $\left(15-30^{\circ} \mathrm{C}\right)$ favor fungal activity [14]. Spore germination and cuticle penetration occurred on leaflets of peanut and the symptoms appeared seven to 9 days after inoculation. The initial symptoms of small, irregular, brown to reddish brown lesions along the midrib of both young and old leaves were observed and the severe defoliation was observed in the infected fields of susceptible cultivars [15]. Following penetration of the cuticle, fungal hyphae grew just under the cuticle for a few $\mathrm{mm}$ beyond the point of penetration and ceased growth, which indicated that the defense responses of $P$. araehidieola infection is hypersensitive-type reaction [6].

Although the web blotch disease has considerable economic importance in peanut farming systems, previous researches on this disease are relatively few compared with those on other main peanut foliar diseases. Zhang et al. (2019) presented the draft genome sequence of a Phoma araehidieola isolate named $\mathrm{Wb} 2$ and indicated that the draft genome of $\mathrm{Wb} 2$ was about $34.11 \mathrm{Mb}$ and contained 37,330 open reading frames (ORFs), with G + C content 49.23\% [16]. Smith et al. (1979) reported that Virginia and runner market-type peanut cultivars are more resistant to web blotch than the Spanish markettype cultivars [17]. Zhang et al. (2011) showed that resistance to web blotch was controlled by three major genes and several minor genes, and identified one quantitative trait locus (QTL) located on linkage group (LG) 7 [18]. At present, researches on peanut web blotch mainly focus on the classification status of the pathogen asexual generation, the pathogen molecular biology, chemical control and epidemic rules $[6-9,14,15]$. A similar disease called net blotch, caused by Pyrenophora teres is also one of the major diseases in barley [19]. Many studies have identified a large region of chromosome $6 \mathrm{H}$ responsible for resistance to net blotch in barley [20-23] and a region in chromosome $3 \mathrm{H}$ harbors two predicted genes from the NBS-LRR gene family [24].

With advances in genomic sequencing technologies and the availability of diploid and tetraploid genome assemblies in Arachis species [1, 4], high-throughput genome-wide sequencing has become a primary strategy in peanut to identify single nucleotide polymorphisms (SNP) markers linked to resistance genes and QTLs. For example, four bacterial wilt resistance QTLs were identified on chromosome B02 using a RIL population and 2187 SNP markers [25]. A major QTL for resistance to late leaf spot, on chromosome B05, and two major QTLs for resistance to early leaf spot, on chromosomes A03 and B04, were mapped using a high-density genetic map comprising 2753 SNP markers and a $F_{9}$ RIL population of 192 individual lines [26]. Additionally, 62 QTLs for 14 yield-related traits were detected on 12 chromosomes across three environments using a high-density genetic map including 2636 recombination bin markers and a $F_{6}$ RIL population of 242 lines [27].

In the present study, high-throughput genome-wide sequencing technology was used to obtain SNP markers, and a SNP-based genetic linkage map was constructed to identify the QTLs for resistance to peanut web blotch. The results of this study will help to better understand the mechanisms of interaction between $P$. araehidieola and peanut and to develop resistant varieties.

\section{Results}

\section{Evaluation of web blotch resistance}

Wide phenotypic variations in response to peanut web blotch were observed among RILs under all the five test environments in this study and the two parental varieties of the RIL population also showed significant difference in web blotch resistance (Fig. 1). The distributions of the disease scale recorded during the years 2007 and 2008 were shown in Fig. 1a, whereas the distributions of the disease index recorded during the years 2012 and 2018 (both in the field and indoor) were shown in Fig. 1b-d. The frequency distribution of disease index in the year 2012 was almost a normal distribution (Fig. 1b). The frequency distributions under other conditions displayed skewed distribution (Fig. 1a, c and d), which had no impact on the following mapping results because only the random error term of the phenotypic data was required to follow the normal distribution [28].

\section{Sequencing, SNP and bin markers discovery}

A whole-genome resequencing strategy was applied to construct paired-end libraries for the parental lines and their 212 RIL progenies. The length of DNA fragments in the libraries was about $350 \mathrm{bp}$. Approximately, 490 $\mathrm{Gb}$ of clean data $(\mathrm{Q} 20>96 \%)$ were produced, resulting from 6285 million reads, each with a length of $150 \mathrm{bp}$. In total, 600 million reads were generated for each of the two parents, whereas reads generated for each of the RILs varied from 22.37 to 24.83 million (Supplementary Table S1). Coverage rate, mapped reads rate, sequence depth and other results from alignment to the reference genome were shown in Supplementary Table S1. In particular, the coverage rate associated with the two parents Zheng8903 and Yuhua4 were 98.51 and $99.05 \%$, respectively, whereas it ranged from 53 to $63.63 \%$ in the RIL population (Supplementary Table S1). The sequencing depth was $35.23 \times$ for both parents, whereas it ranged from $1.31 \times$ to $1.46 \times$ for the RIL population. Originally, 636,831 SNPs were called from the 214 samples using 

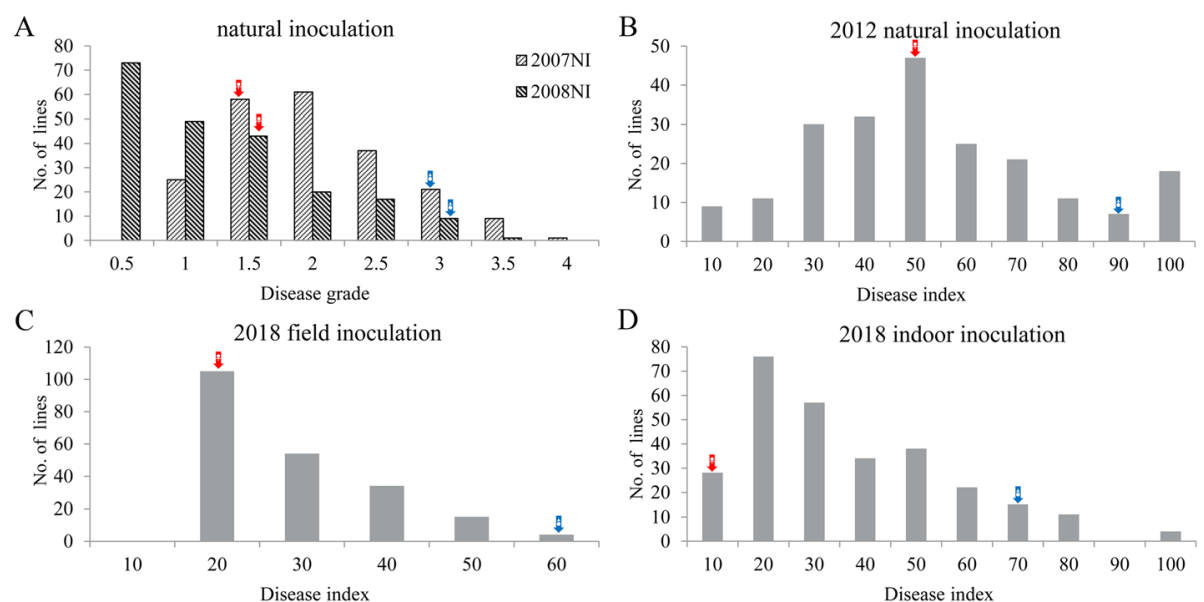

Fig. 1 Response of RILs to peanut web blotch in five experimental trials. Red and blue arrows stand for the positons of resistant (female) and susceptible (male) parents respectively. a. The frequency distribution of disease grade under natural inoculation in the year 2007 and 2008 . b. The frequency distribution of disease index under natural inoculation in the year 2012. c. The frequency distribution of disease index under field inoculation in the year 2018. d. The frequency distribution of disease index under indoor inoculation in the year 2018

the GATK protocol. Then, 556,615 SNPs were retained after filtering for low quality loci in the two parents, due to missing values, heterozygosis, depth $<10$ and $\mathrm{GQ}<$ 20. Finally, 138,039 SNPs which were homozygous and polymorphic between two parents were used for further analyses.

\section{Construction of physical recombination maps and high density genetic linkage map}

To avoid errors caused by low coverage associated with RIL sequencing, a sliding window with 15 consecutive SNPs was used to find the more accurate recombined breakpoints. The physical recombination map of 212 RILs was constructed based on the recombination map of each progeny (Supplementary Figure S1). After that, all chromosomes of the 212 RILs were aligned and compared for the minimal of $100-\mathrm{kb}$ intervals. As a result, a total of 3634 bin markers for the 212 lines were obtained in this way, and the genotypes and physical locations of the bins were given in Supplementary Table S2.

The obtained 3634 bin markers were used to construct a genetic linkage map by the software JoinMap ${ }^{\circ} 5.0$ [29]. Twenty linkage groups were generated and assigned to the 20 chromosomes of the cultivated peanut according to the physical positions. The total genome length was $1817.91 \mathrm{cM}$ and the marker density across the 20 linkage groups ranged from 0.39 to $0.66 \mathrm{cM}$ with an average of $0.50 \mathrm{cM}$ (Table 1). The LG16 had the lowest marker number (129) and the shortest genetic length (54.58 cM), while LG3 had the highest marker number (277) and the longest genetic length $(135.61 \mathrm{cM})$ (Table 1 and Fig. 2). More than $97.5 \%$ of the inter-markers distances were lower than $3 \mathrm{cM}$. The highest inter-marker distance $(16.06 \mathrm{cM})$ was associated with LG6 (Table 1).
QTL mapping and candidate genes prediction for peanut web blotch resistance

QTL mapping of peanut web blotch resistance was performed with MapQTL ${ }^{\circ}$ v6.0 [30], using phenotypic data collected across five environments. Eight QTLs associated with peanut web blotch resistance, located in eight different LGs, were confirmed in at least two environments, explaining from 2.8 to $15.1 \%$ of phenotypic variation and displaying LOD values ranging from 1.32 to 7.45 (Table 2). Two QTLs ( $q$ WBRA04 and $q$ WBRA14) located on LG04 and LG14 were significantly associated with resistance in all the five testing environments in this study (Table 2, Fig. 3 and Fig. 4) and explained more than $10 \%$ of phenotypic variation, indicating they are probably the major QTLs with stable expression. Except for $q W B R A 13$ and $q W B R A 05$, which were detected in four and two environments respectively, the other four QTLs qWBRA03, qWBRA16, qWBRA17, qWBRA19 were detected in three environments (Table 2). Absolute values displayed by the additive effect ranged from 0.11 to 8.29, and were negative for the QTLs on LG4, LG5, LG13, LG14, LG19 (indicating that the favorable alleles originated from the resistant parent Zheng8903), and positive for the QTLs on LG3, LG16 and LG17 (indicating that the favorable alleles originated from the susceptible parent Yuhua4).

To identify candidate genes for peanut web blotch resistance, coding sequences in the genomic region associated with the QTLs qWBRA04 and qWBRA14 were examined for predicted function, according to the Arachis hypogaea cv. Tifrunner reference genome annotation database [4]. A total of 40 candidate genes were identified with a putative role in disease resistance (Table 3). In detail, the region of $q W B R A 04$, spanning a 
Table 1 Summary information on the 20 linkage groups (LGs) detected in this study

\begin{tabular}{|c|c|c|c|c|c|}
\hline ID & Total markers & Total distance (cM) & Average distance (cM) & Max inter-marker distance (cM) & Inter-markers distances $<=3 \mathrm{cM}$ \\
\hline LG1 & 164 & 94.98 & 0.58 & 4.26 & $98.78 \%$ \\
\hline LG2 & 152 & 83.88 & 0.55 & 2.27 & $100.00 \%$ \\
\hline LG3 & 277 & 135.61 & 0.49 & 2.54 & $100.00 \%$ \\
\hline LG4 & 168 & 111.21 & 0.66 & 6.33 & $98.21 \%$ \\
\hline LG5 & 194 & 75.76 & 0.39 & 3.96 & $99.48 \%$ \\
\hline LG6 & 200 & 129.82 & 0.65 & 16.06 & $97.50 \%$ \\
\hline LG7 & 170 & 88.20 & 0.52 & 2.54 & $100.00 \%$ \\
\hline LG8 & 210 & 109.86 & 0.52 & 5.35 & $99.52 \%$ \\
\hline LG9 & 192 & 94.31 & 0.49 & 2.82 & $100.00 \%$ \\
\hline LG10 & 160 & 75.41 & 0.47 & 1.74 & $100.00 \%$ \\
\hline LG11 & 129 & 61.81 & 0.48 & 3.39 & $99.22 \%$ \\
\hline LG12 & 176 & 72.90 & 0.41 & 1.74 & $100.00 \%$ \\
\hline LG13 & 240 & 110.15 & 0.46 & 2.41 & $100.00 \%$ \\
\hline LG14 & 189 & 82.88 & 0.44 & 2.14 & $100.00 \%$ \\
\hline LG15 & 234 & 91.81 & 0.39 & 2.41 & $100.00 \%$ \\
\hline LG16 & 129 & 54.58 & 0.42 & 2.27 & $100.00 \%$ \\
\hline LG17 & 178 & 97.78 & 0.55 & 2.68 & $100.00 \%$ \\
\hline LG18 & 143 & 74.99 & 0.52 & 3.39 & $98.60 \%$ \\
\hline LG19 & 182 & 96.90 & 0.53 & 3.39 & $98.90 \%$ \\
\hline LG20 & 147 & 75.07 & 0.51 & 3.67 & $99.32 \%$ \\
\hline Total & 3634 & 1817.91 & 0.50 & & \\
\hline
\end{tabular}

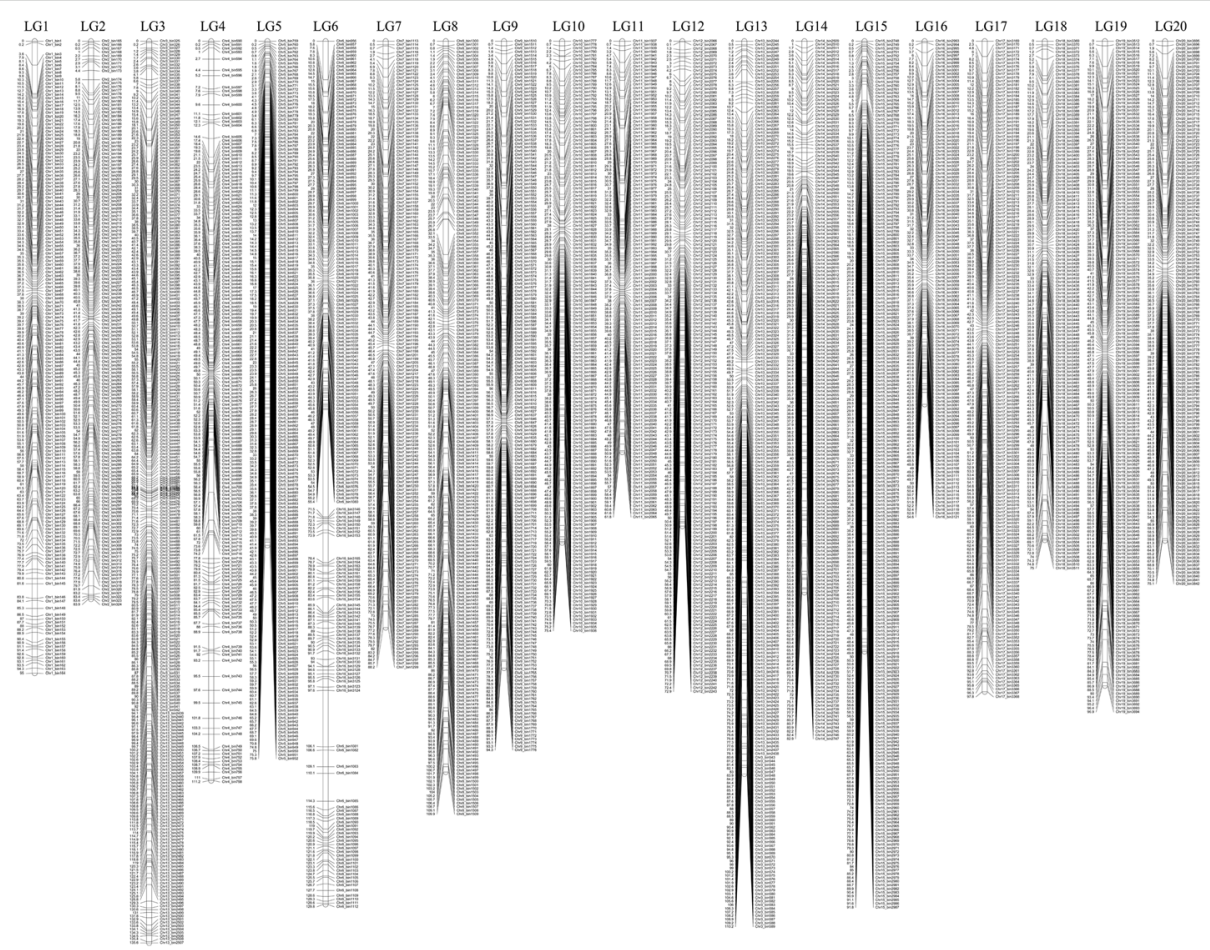

Fig. 2 Genetic map obtained from the Zheng $8903 \times$ Yuhua4 RIL population 
Table 2 Quantitative trait loci (QTLs) for peanut web blotch resistance identified in this study

\begin{tabular}{llllllll}
\hline QTL & LG & Position (cM) & Left-Right Marker & LOD & PVE\% & Add effects & Environment \\
\hline 9WBRA03 & LG03 & 29.84 & Chr3_bin366-Chr3_bin367 & $3.48 \sim 5.37$ & $7.4 \sim 11.0$ & $0.21 \sim 5.85$ & $2007,2008,2012$ \\
9WBRA04 & LG04 & 110.17 & Chr4_bin756-Chr4_bin757 & $2.35 \sim 7.45$ & $5.0 \sim 15.1$ & $-8.29 \sim-0.15$ & $2007,2008,2012,2018^{\mathrm{a}}, 2018^{\mathrm{b}}$ \\
9WBRA05 & LG05 & 0.48 & Chr5_bin760-Chr5_bin761 & $2.88 \sim 3.28$ & $6.9 \sim 8.8$ & $-6.1 \sim-5.61$ & $2012,2018^{\mathrm{b}}$ \\
9WBRA13 & LG13 & 64.06 & Chr13_bin2385-Chr13_bin2386 & $1.88 \sim 3.86$ & $4.0 \sim 8.0$ & $-5.32 \sim-0.15$ & $2007,2008,2012,2018^{\mathrm{a}}$ \\
9WBRA14 & LG14 & 82.88 & Chr14_bin2746-Chr14_bin2747 & $1.32 \sim 4.08$ & $2.8 \sim 11.2$ & $-6.9 \sim-.011$ & $2007,2008,2012,2018^{\mathrm{a}}, 2018^{\mathrm{b}}$ \\
qWBRA16 & LG16 & 41.84 & Chr16_bin3084-Chr16_bin3085 & $2.16 \sim 3.18$ & $5.5 \sim 6.7$ & $0.18 \sim 5.51$ & $2008,2012,2018^{\mathrm{b}}$ \\
9WBRA17 & LG17 & 45.25 & Chr17_bin3253-Chr17_bin3254 & $3.33 \sim 4.98$ & $7.0 \sim 10.2$ & $0.21 \sim 6.83$ & $2008,2012,2018^{\mathrm{a}}$ \\
9WBRA19 & LG19 & 94.09 & Chr19_bin3690-Chr19_bin3691 & $1.93 \sim 3.03$ & $4.1 \sim 6.4$ & $-2.22 \sim-0.13$ & $2007,2008,2018^{\mathrm{a}}$
\end{tabular}

$2018^{\mathrm{a}}$ stands for field, $2018^{\mathrm{b}}$ stands for indoor

linkage interval of $1.10 \mathrm{cM}$, corresponds to a physical interval of $\sim 86 \mathrm{~kb}$ and contains four nucleotide binding site-leucine rich repeat (NBS-LRR) genes. The genes Arahy.Q7VTCQ and Arahy.9YX67Z contain coiled-coil (CC) domains and the genes Arahy.SK6LYR and Arahy.1RZOPJ contain Toll-interleukin receptor (TIR) domain (Table 3). The region of $q W B R A 14$, spanning 0.48 $\mathrm{cM}$, physically corresponds to $\sim 2.8 \mathrm{Mb}$ and contains 37 genes encoding disease resistance protein. Among them, 19 genes contain TIR domains and one gene contains CC domain, whereas the remaining 17 genes encode other proteins with a putative role in disease resistance, such as a $b Z I P$ transcription factor and a WRKY transcription factor-like protein.

To further validate the candidate genes, the mutation type of the SNPs located in the candidate genes were analyzed (Table 4). There were 24 polymorphic SNPs between two parents located in seven genes, eight of which were in noncoding DNA regions and two of which were synonymous mutation, and the remained 14 SNPs on chromosome A03, A04, and A14 were non-synonymous. The 14 non-synonymous SNPs were located in four coding sequence regions, four of which were in the region of Arahy.LFEOTK, one in Arahy.Q7VTCQ, three in

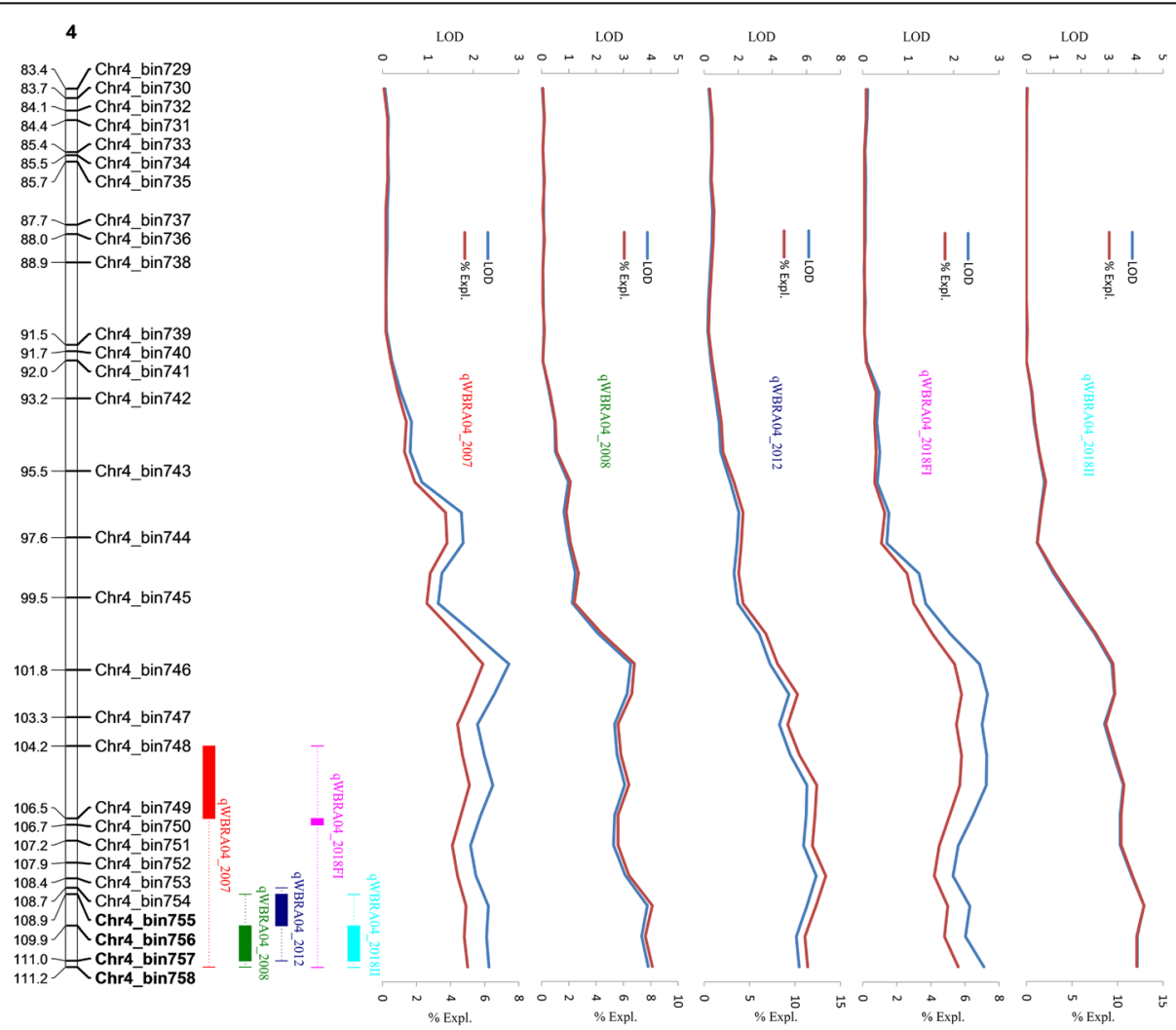

Fig. 3 Position, LOD and PVE (\%Expl.) curves of the QTL detected on LG4 
14

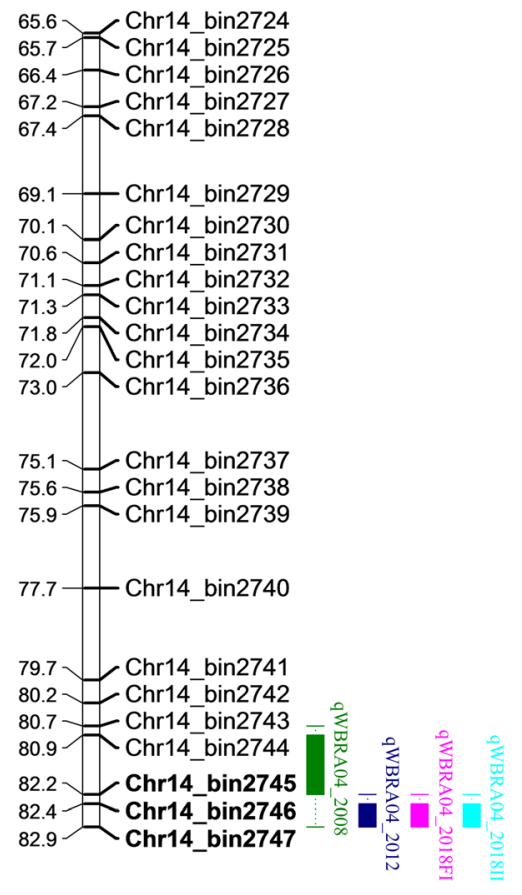

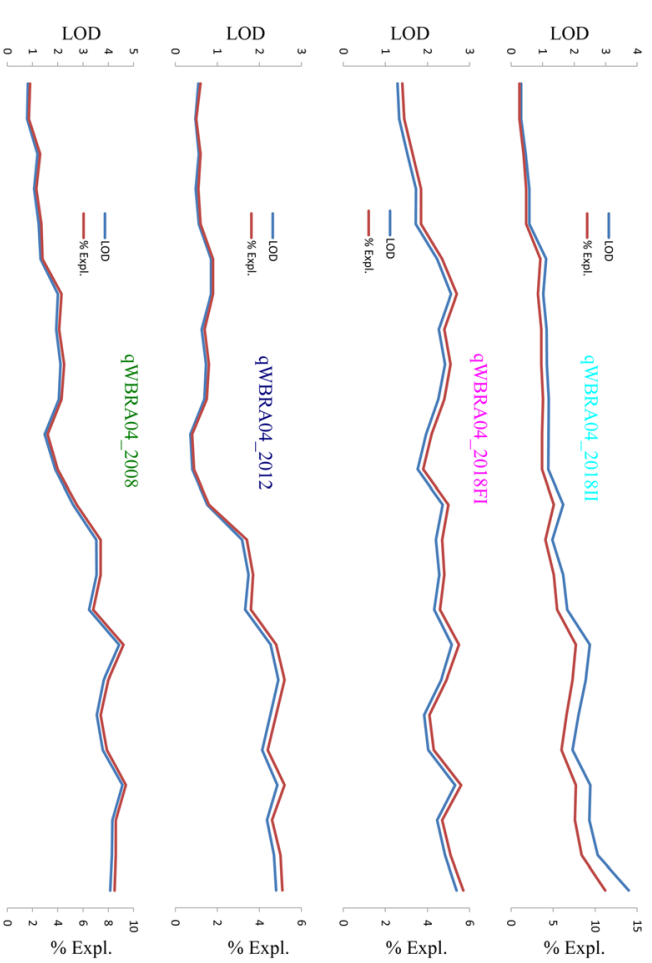

Fig. 4 Position, LOD and PVE (\%Expl.) curves of the QTL detected on LG14

Arahy.1RZOPJ and six in Arahy.MR7539. All of the four genes were NBS-LRR, and three of them contained TIR domain except that Arahy.Q7VTCQ contained CC domain.

\section{KASP design and validation}

To realize molecular marker assisted breeding for web blotch resistant peanut varieties, the 24 polymorphic SNPs between two parents (Table 4) were used to develop KASP (Kompetitive allele specific PCR) markers which were validated using 47 lines (23 resistant lines and 24 susceptible lines) from this population. Results indicated that three SNPs on chromosome A04 in the region of Arahy.Q7VTCQ might be related to peanut web blotch resistance (Supplementary Table S3). Most of the tested lines (42 out of 47 lines) showed consistency between their phenotypes and genotypes in different test conditions. Two susceptible genotypes (WB6235 and WB6360) that showed low level of infection in some conditions, probably escaped from inoculation (Supplementary Table S3). One susceptible line (WB6204) and two resistant lines (WB6248 and WB6367) were inconsistent with their phenotypes (Supplementary Table S3).

\section{Discussion}

A high density genetic linkage map with 3634 bin markers was constructed based on high-throughput whole-genome sequencing and a sliding window strategy. The order of the markers on the genetic map was overall consistent with the physical order according to the peanut genome assembly, except for some translocation occurring between LG3 and LG13 and between LG6 and LG16. A total of 277 bin markers were distributed on LG3, while the physical positions of the last 70 markers were on chromosome A13, and the physical positions of the last 47 markers of LG13 were on chromosome A03. Moreover, 43 bin markers from chromosome A16 were inserted in the LG6. The reason for such seeming translocation might be that there were some assembly errors in the reference genome which were illustrated in Peanutbase (https://www.peanutbase. org/data/public/Arachis_hypogaea/Tifrunner.gnm1.KYV3/) [31]. The genetic orders of the markers on LG1, LG4, LG5, LG10, LG12 and LG18 were fully consistent with their physical orders and a few markers on the other LGs were inversed with the adjacent markers.

In total, eight novel QTLs distributed on eight chromosomes were identified for peanut web blotch resistance, which were confirmed in at least two of the experimental trials. Three of them were major QTLs, as they were associated with phenotypic variance explained $(\mathrm{PVE})>10 \%$, whereas the other five were minor QTLs, in accordance with the predicted results of Zhang et al. (2011). Except for qWBRA03, which was detected only in naturally conditions, the 
Table 3 Candidate genes for peanut web blotch resistance in the region of qWBRA04 and qWBRA14

\begin{tabular}{|c|c|c|}
\hline Gene model & Gene Location & Annotation \\
\hline Arahy.Q7VTCQ & Arahy.04:125904648-125,913,229 & CC-NBS-LRR \\
\hline Arahy.SK6LYR & Arahy.04:125919867-125,922,353 & TIR-NBS-LRR \\
\hline Arahy.9YX67Z & Arahy.04:125933796-125,935,754 & CC-NBS-LRR \\
\hline Arahy.1RZOPJ & Arahy.04:125978283-125,982,796 & TIR-NBS-LRR \\
\hline Arahy.MR7539 & Arahy.14:140429517-140,433,390 & TIR-NBS-LRR \\
\hline Arahy.5JS28N & Arahy. $14: 140673363-140,675,200$ & TIR-NBS-LRR \\
\hline Arahy.098P1D & Arahy. $14: 140716472-140,720,198$ & TIR-NBS-LRR \\
\hline Arahy.B7IYCZ & Arahy. $14: 140737488-140,743,003$ & TIR-NBS-LRR \\
\hline Arahy.GQ7H4X & Arahy.14:140748678-140,752,470 & TIR-NBS-LRR \\
\hline Arahy.KRT76W & Arahy.14:140775690-140,779,279 & TIR-NBS-LRR \\
\hline Arahy.ARVN7W & Arahy. 14:140932228-140,936,295 & Ethylene-responsive transcription factor $1 \mathrm{~B}$ \\
\hline Arahy.QC7JPT & Arahy.14:140964016-140,967,594 & MYB/SANT-like DNA-binding domain protein \\
\hline Arahy.ULYV8E & Arahy.14:141015920-141,031,728 & E3 ubiquitin protein ligase DRIP2-like \\
\hline Arahy.M6XMZZ & Arahy. 14:141138899-141,141,424 & Disease resistance protein \\
\hline Arahy.GF38LY & Arahy. 14:141242234-141,244,304 & TIR-NBS-LRR \\
\hline Arahy.Z4PSSR & Arahy.14:141280146-141,281,153 & TIR-NBS-LRR \\
\hline Arahy.38PKYC & Arahy.14:141292017-141,295,699 & TIR-NBS-LRR \\
\hline Arahy.3HI1A9 & Arahy.14:141393957-141,398,159 & TIR-NBS-LRR \\
\hline Arahy.6V6NN7 & Arahy. $14: 141400695-141,406,643$ & TIR-NBS-LRR \\
\hline Arahy.1B47W & Arahy.14:141409110-141,411,145 & LRR and NB-ARC domain disease resistance protein \\
\hline Arahy.UDNB1T & Arahy.14:141411916-141,413,951 & LRR and NB-ARC domain disease resistance protein \\
\hline Arahy.1VN7JI & Arahy. 14:141416418-141,422,328 & TIR-NBS-LRR \\
\hline Arahy.D05D8X & Arahy.14:141438610-141,439,200 & Disease resistance protein RPP13-like \\
\hline Arahy.5TMH4E & Arahy.14:141431509-141,437,763 & TIR-NBS-LRR \\
\hline Arahy.NA6KD0 & Arahy. $14: 141445227-141,450,684$ & TIR-NBS-LRR \\
\hline Arahy.WD8J75 & Arahy. 14:141458141-141,462,928 & TIR-NBS-LRR \\
\hline Arahy.UZFH7Q & Arahy. 14:141463237-141,467,397 & TIR-NBS-LRR \\
\hline Arahy.5MNM78 & Arahy.14:141468339-141,471,341 & TIR-NBS-LRR \\
\hline Arahy.388Y5C & Arahy.14:141508791-141,512,359 & TIR-NBS-LRR \\
\hline Arahy.M9HGBQ & Arahy.14:141514206-141,520,849 & bZIP transcription factor \\
\hline Arahy.BJT98M & Arahy.14:141539637-141,544,390 & WRKY transcription factor-like protein \\
\hline Arahy.EDAK6K & Arahy.14:141674291-141,674,683 & CC-NBS-LRR \\
\hline Arahy.RZ4S3T & Arahy.14:141672176-141,674,272 & Disease resistance protein \\
\hline Arahy.QH59CH & Arahy.14:141683064-141,685,622 & Disease resistance protein \\
\hline Arahy.GM4BOI & Arahy.14:141689106-141,691,574 & Disease resistance protein \\
\hline Arahy.U06TWT & Arahy.14:141696720-141,699,293 & Disease resistance protein \\
\hline Arahy.8161S0 & Arahy.14:141708397-141,711,939 & Disease resistance protein \\
\hline Arahy.ZY30Q0 & Arahy. 14:141719033-141,719,888 & Disease resistance protein \\
\hline Arahy.N7WF48 & Arahy.14:141789316-141,794,004 & TCP7 transcription factor \\
\hline Arahy.QA8QB9 & Arahy.14:143064164-143,068,579 & E3 ubiquitin-protein ligase \\
\hline
\end{tabular}

other seven QTLs were detected both in the natural and artificial inoculation conditions. The two major QTLs $q$ WBRA04 and $q$ WBRA14 were stably detected across all the five testing environments in this study, and thus might be of great potential in breeding resistant peanut varieties.

In the present study, we identified 55 candidate disease resistant genes in the target region of eight QTLs 
Table 4 The mutation type of the SNPs located in the candidate genes

\begin{tabular}{|c|c|c|c|c|c|}
\hline Gene model & Chromosome & Position & Resistant parent allele & Susceptible parent allele & Mution type \\
\hline \multirow[t]{5}{*}{ Arahy.LFEOTK } & Arahy.03 & $5,689,199$ & A & $\mathrm{T}$ & noncoding DNA regions \\
\hline & Arahy.03 & $5,689,634$ & $\mathrm{~T}$ & $C$ & $\mathrm{~T} \rightarrow \mathrm{C}, \mathrm{Glu} \rightarrow$ Gly \\
\hline & Arahy.03 & $5,692,237$ & A & G & $\mathrm{A} \rightarrow \mathrm{G}, \mathrm{Cys} \rightarrow \operatorname{Arg}$ \\
\hline & Arahy.03 & $5,694,410$ & $C$ & $\mathrm{~T}$ & $\mathrm{C} \rightarrow \mathrm{T}, \mathrm{Arg} \rightarrow \mathrm{His}$ \\
\hline & Arahy.03 & $5,696,415$ & A & C & $\mathrm{A} \rightarrow \mathrm{C}, \mathrm{Ser} \rightarrow$ Ala \\
\hline \multirow[t]{5}{*}{ Arahy.Q7VTCQ } & Arahy.04 & $125,905,992$ & $\mathrm{~T}$ & C & noncoding DNA regions \\
\hline & Arahy.04 & $125,907,949$ & A & G & noncoding DNA regions \\
\hline & Arahy.04 & $125,909,638$ & $C$ & $\mathrm{~T}$ & noncoding DNA regions \\
\hline & Arahy.04 & $125,911,313$ & G & $\mathrm{T}$ & Synonymous mutation, Ala \\
\hline & Arahy.04 & $125,912,343$ & G & $\mathrm{T}$ & $\mathrm{G} \rightarrow \mathrm{T}, \mathrm{Asp} \rightarrow \mathrm{Tyr}$ \\
\hline \multirow[t]{3}{*}{ Arahy.1RZOPJ } & Arahy.04 & $125,980,437$ & $C$ & G & $\mathrm{C} \rightarrow \mathrm{G}, \mathrm{Thr} \rightarrow \mathrm{Ser}$ \\
\hline & Arahy.04 & $125,981,121$ & A & C & $\mathrm{A} \rightarrow \mathrm{C}$, Lys $\rightarrow \mathrm{Thr}$ \\
\hline & Arahy.04 & $125,981,989$ & A & C & $A \rightarrow C$, Lys $\rightarrow$ Asp \\
\hline Arahy.00XS3D & Arahy.05 & $7,865,324$ & $\mathrm{~T}$ & G & noncoding DNA regions \\
\hline Arahy.IY6P5S & Arahy.05 & $8,315,940$ & T & $C$ & noncoding DNA regions \\
\hline \multirow[t]{7}{*}{ Arahy.MR7539 } & Arahy.14 & $140,432,094$ & G & C & $\mathrm{G} \rightarrow \mathrm{C}$, Lys $\rightarrow$ Asp \\
\hline & Arahy.14 & $140,432,405$ & T & A & $\mathrm{T} \rightarrow \mathrm{A}, \mathrm{Phe} \rightarrow \mathrm{Tyr}$ \\
\hline & Arahy.14 & $140,432,499$ & T & G & Synonymous mutation, Val \\
\hline & Arahy.14 & $140,432,542$ & G & C & $\mathrm{G} \rightarrow \mathrm{C}, \mathrm{Val} \rightarrow \mathrm{Leu}$ \\
\hline & Arahy.14 & $140,432,546$ & T & G & $\mathrm{T} \rightarrow \mathrm{G}, \mathrm{Val} \rightarrow \mathrm{Gly}$ \\
\hline & Arahy.14 & $140,432,725$ & G & $\mathrm{T}$ & $\mathrm{G} \rightarrow \mathrm{T}$, Gly $\rightarrow$ Cys \\
\hline & Arahy.14 & $140,432,848$ & G & C & $\mathrm{G} \rightarrow \mathrm{C}, \mathrm{Glu} \rightarrow \mathrm{Gln}$ \\
\hline \multirow[t]{2}{*}{ Arahy.NC1Z37 } & Arahy.17 & $26,849,446$ & C & $\mathrm{T}$ & noncoding DNA regions \\
\hline & Arahy.17 & $26,855,030$ & C & $\mathrm{T}$ & noncoding DNA regions \\
\hline
\end{tabular}

(Tables 3 and 5), of which 40 were linked with the two major and stable QTLs $q$ WBRA04 and qWBRA14 (Table $3)$, while the other 15 were associated with the other six QTL intervals (Table 5). The 40 candidate genes covered by the intervals of $q$ WBRA04 and $q$ WBRA14 included 21 TIR-NBS-LRR and 3 CC-NBS-LRR, which are the two well-known $R$ gene types [32]. Also, there were two candidate genes encoded LRR and NB-ARC (nucleotidebinding domain shared with APAF-1, various R-proteins and CED-4) domain, which also encoded resistance genes [33]. Among the remained 15 genes, seven of them function in the downstream pathways of resistant signaling, the other eight encoded proteins contain disease resistance response related domains but could not assigned to the well-known R-gene types (Table 3).

A total of 26 NBS-LRR genes were identified to be related to peanut web blotch resistance in this study. NBSLRR is the biggest category of R genes [34] and has been identified at the genome-wide level in Arachis [35]. It had been found that NBS-LRRs were involved in response to late leaf spot, tomato spotted wilt virus, and bacterial wilt in A. duranensis, A. ipaensis, and A. hypogaea [36]. It could be concluded that NBS-LRR was also involved in the resistance to peanut web blotch, but the regulatory mechanism in the process of disease resistance needs to be further studied.

The results of three validated KASP markers indicated that the gene Arahy.Q7VTCQ (CC-NBS-LRR) might be one of the resistant genes for peanut web blotch in a great possibility. The reason of the inconsistence between the phenotypes and genotypes of three test lines may be that the three KASP markers were not completely linked with peanut web blotch resistance. Therefore, further study will be needed to design closer linked markers to be employed in molecular marker assisted breeding (MAS).

\section{Conclusion}

In this study, eight QTLs for peanut web blotch resistance were detected and two major QTLs qWBRA04 and qWBRA04 were linked to 40 candidate genes encoding NBS-LRR or other proteins related to disease resistance, which may shed some insights on understanding web blotch resistance and facilitate the development of resistant peanut cultivars. 
Table 5 Candidate genes for peanut web blotch resistance in the region of six QTLs identified in this study

\begin{tabular}{|c|c|c|}
\hline Gene model & Gene Location & Annotation \\
\hline Arahy.LFEOTK & Arahy.03:5688642-5,697,084 (- strand) & TIR-NBS-LRR \\
\hline Arahy. $25 \mathrm{H} 20 \mathrm{~J}$ & Arahy.03:5707178-5,707,681 (- strand) & TIR-NBS-LRR \\
\hline Arahy.FHP2K2 & Arahy.03:5719557-5,720,120 (+ strand) & Disease resistance-responsive protein \\
\hline Arahy.PQJ7DP & Arahy.03:5791188-5,792,666 (+ strand) & MYB transcription factor \\
\hline Arahy.00XS3D & Arahy.05:7862831-7,866,802 (+ strand) & Ethylene-responsive transcription factor 3 \\
\hline Arahy.S3U17M & Arahy.05:8182461-8,184,440 (- strand) & MYB family transcription factor \\
\hline Arahy.90DV49 & Arahy.05:8258615-8,262,245 (+ strand) & Transcription factor SPATULA-like(bHLH) \\
\hline Arahy.IY6P5S & Arahy.05:8314264-8,318,032 (+ strand) & Protein kinase superfamily protein \\
\hline Arahy.DIZB8V & Arahy.13:43668419-43,670,419 (+ strand) & Ethylene-responsive transcription factor 3 \\
\hline Arahy.0E1GBK & Arahy. 13:44877348-44,878,744 (- strand) & ZIP zinc/iron transport family protein \\
\hline Arahy.8JT992 & Arahy.13:45354167-45,355,323 (- strand) & MYB transcription factor \\
\hline Arahy.2NFP2H & Arahy.19:157562932-157,564,981 (+ strand) & Heat shock transcription factor A2 \\
\hline Arahy.5KF5UG & Arahy.19:157602650-157,604,922 (- strand) & bZIP transcription factor family protein \\
\hline Arahy.ZMEP2D & Arahy. 16:121662983-121,668,124 (+ strand) & HSP20-like chaperones superfamily protein \\
\hline Arahy.NC1Z37 & Arahy. 17:26845330-26,857,334 (- strand) & bZIP transcription factor family protein \\
\hline
\end{tabular}

\section{Methods}

\section{Plant materials}

A RIL population consisting of $212 \mathrm{~F}_{12}$ lines derived from the parental cross combination between lines Zheng8903 and cultivar Yuhua4 was used in this study. The female parent Zheng8903 with the pedigree '79-266//71-31/Chico' is a breeding line showing high level of resistance to peanut web blotch [37]. The male parent Yuhua4 is a variety released in 1991 by the Henan Academy of Agricultural Sciences and we obtained the seeds from our own inventory [38], showing high level of susceptibility to web blotch [37]. All the plant materials including RILs and its parents mentioned above were developed and preserved in the corresponding author's lab.

\section{Experimental trials and phenotyping}

All the experimental trials to evaluate response to peanut web blotch were conducted at the research station of the Henan Academy of Agricultural Science from May to September. Plant materials mentioned above were evaluated following natural infection, in field trials carried out in 2007, 2008 and 2012, and following artificial inoculation, in field and indoor trials carried out in 2018. For field trails, twenty seeds for each genotype were sown in $3 \mathrm{~m}$ long and $0.4 \mathrm{~m}$ wide plots, according to a complete random block design with two replicates for natural infection and three replicates for artificial inoculation. For natural infection in the year 2007, 2008 and 2012, disease evaluation was carried out before harvest according to the $0-4$ scale described in Yuan et al. (2004) [39]. For field inoculation in the year 2018, plots were sprayed with an inoculum of $1.6 \times 10^{-3} \mathrm{~g} / \mathrm{ml}$ at the flowering stage [40] and disease evaluation was carried out 20 days after inoculation according to the $0-9$ scale described in $\mathrm{Yu}$ (2011) [41]. For indoor inoculation, five plants were inoculated for each line with two replications at the 6 leaves stage. The inoculum concentration was $2 \times 10^{6}$ conidia/ml and the preparation of conidia suspension described by Zhang (2019) [37] was followed. Two weeks after inoculation, 12 inoculated leaves at the main stem were collected and the lesion area for each was scanned by the Leaf Area Meter (Wanshen LA-S). The indoor classification standard of peanut web blotch was as follows: for scale 0 , no lesion detected; scale $1,0<$ lesion area $<6 \%$; scale $2,6 \% \leq$ lesion area $<25 \%$; scale 3 , $25 \% \leq$ lesion area $<50 \%$; scale $4,50 \% \leq$ lesion area $<75 \%$; scale $5,75 \% \leq$ lesion area.

\section{Sequencing and genotyping}

Genomic DNA of the parents and 212 RILs was extracted from young leaf tissues using the Plant genome DNA extraction kit (TIANGEN) and randomly sheared by sonication. The DNA fragments with the length of $300 \mathrm{bp}$ were recovered by electrophoresis. After ligating DNA fragments with adapters, libraries were paired-end sequenced using the Illumina Hi-seq platform with read length of $150 \mathrm{bp}$.

After trimming adapters and low quality reads, clean data was used for aligning to the reference genome, allowing SNP identification and genotyping. In detail, the assembly of Arachis hypogaea cv. Tifrunner was used as the reference genome [4]. The aln command in the software bwa-0.7.10 was used to align clean data to the reference genome, and unique reads were used for subsequent SNP variation detection by the software 
GATK3.3.0. The obtained SNP sets of two parents were filtered used the missing values, heterozygosis, depth and GQ value and the homozygous and polymorphic loci were used for the RIL population. The binary alignment mapping (BAM) files obtained in this study have been submitted to the BioProject database at NCBI under the BioProject ID: PRJNA602098.

\section{Linkage map construction and QTL mapping}

As the accuracy of call at single SNP loci was low, due to the low coverage chosen for RIL sequencing, a sliding window approach was applied to evaluate a group of consecutive SNPs for genotyping [42]. The genotypes of all chromosomes of the 212 RILs were aligned and compared for the minimal of $100-\mathrm{kb}$ intervals and the adjacent 100-kb intervals with the same genotype across the entire RIL population were recognized as a single recombination bin [42]. From bin markers, linkage groups were constructed using JoinMap v5.0 [29], selecting LOD scores from 2 to 10 to identify groups and the regression algorithm to perform ordering within each LG. The final linkage map was drawn using the $R$ package LinkageMapView. QTL mapping was performed using MapQTL v6.0 [30], by selecting multiple QTL mapping (MQM) to detect potential QTLs with the LOD threshold of 2.5 in at least one environment.

\section{Supplementary information}

Supplementary information accompanies this paper at https://doi.org/10. 1186/s12870-020-02455-8.

\section{Additional file 1: Table S1. The summary information of the sequencing and alignment results.}

Additional file 2: Figure S1. The physical recombination map of 212 RILs on 20 chromosomes. Blue: the genotype of the resistant parent Zheng8903; Red: the genotype of the susceptible parent Yuhua4; Yellow: the heterozygous genotype; RILs were arranged from the top to the bottom and chromosomes were ranked from left to right.

Additional file 3: Table S2. Genotype and position of the bins in the peanut genetic linkage map obtained in this study. The letter A indicates the genotype of the parental line Zheng8903, and the letter B indicates the genotype of the parental line Yuhua4.

Additional file 4: Table S3. The phenotypes and KASP genotyping results of 47 lines. * stands for missing data.

\begin{abstract}
Abbreviations
QTLs: Quantitative trait loci; RIL: Recombinant inbred line; LG: Linkage group; NBS-LRR: Nucleotide-binding site leucine-rich repeat; ORFs: Open reading frames; SNP: Single nucleotide polymorphisms; CC: Coiled-coil; TIR: Tollinterleukin receptor; NB-ARC: Nucleotide-binding domain shared with APAF1, various R-proteins and CED-4; KASP: Kompetitive allele specific PCR; BAM: Binary alignment mapping; PVE: Phenotypic variance explainedqWBRQtI web blotch resistance; MAS: Marker assisted breeding
\end{abstract}

\section{Acknowledgments}

The authors would like to thank Dr. Stefano Pavan (University of Bari Aldo Moro, Italy) for comments on QTLs analysis and editing the English text of a draft of this manuscript.

\section{Authors' contributions}

$\mathrm{HL}$ and ZS performed the laboratory and field experiments, and wrote the manuscript. LQ, FQ, PD, JX, and ZZ1 performed the genotype analysis and QTLs analysis. ZW, SL, and MG provide help in field inoculation. LZ and YC provide help in indoor inoculation. $\mathrm{SH}, \mathrm{BH}, \mathrm{ZZ2}$, and WD provide help to design the experiments and revise the first draft of the paper. $X Z$ conceived and designed the experiments, facilitated the project, and assisted in manuscript preparation. All authors read and approved the final manuscript.

\section{Funding}

This work was supported by China Agriculture Research System (CARS-13), Henan Provincial Agriculture Research System, China (S2012-5), Fund for Distinguished Young Scholars from Henan Academy of Agricultural Sciences (2019JQ02), and Excellent Young Scholars from Henan Academy of Agricultural Sciences (No. 2018YQ05). In addition, we thank partial funding by National Natural Science Foundation of China (No. 31871663), and Major R\&D and Promotion Projects in Henan, China (No. 182102110137), and Henan Academy of Agricultural Sciences Special Fund for independent innovation (2020ZC13).The funding agencies played no role in the design of the study and collection, analysis, and interpretation of data and in writing the manuscript.

\section{Availability of data and materials}

All data generated or analyzed during this study are included in the manuscript and its Additional file 1, Additional file 2, Additional file 3, and Additional file 4. The BAM files about resequencing data of RILs obtained in this study have been submitted to the BioProject database at NCBI under the BioProject ID: PRJNA602098. The materials used during the current study are available from the corresponding authors.

Ethics approval and consent to participate

Not applicable.

\section{Consent for publication}

Not applicable.

\section{Competing interests}

The authors declare that they have no competing interests.

\section{Author details}

${ }^{1}$ College of Agronomy, Shenyang Agricultural University, Shenyang 110866, PR China. ${ }^{2}$ Industrial Crops Research Institute, Henan Academy of Agricultural Sciences / Key Laboratory of Oil Crops in Huang-Huai-Hai Plains, Ministry of Agriculture and Rural Affairs / Henan Provincial Key Laboratory for Genetic Improvement of Oil Crops, Zhengzhou 450002, PR China. ${ }^{3}$ Institute of Plant Protection, Henan Academy of Agricultural Sciences, Zhengzhou 450002, PR China.

Received: 10 February 2020 Accepted: 21 May 2020

Published online: 03 June 2020

\section{References}

1. Bertioli DJ, Cannon SB, Froenicke L, Huang G, Farmer AD, Cannon EK, Liu X, Gao D, Clevenger J, Dash S, Ren L, Moretzsohn MC, Shirasawa K, Huang W, Vidigal B, Abernathy B, Chu Y, Niederhuth CE, Umale P, Araújo AC, Kozik A, Burrow MD, Varshney RK, Wang X, Zhang X, Barkley N, Guimarães PM, Isobe S, Guo B, Liao B, Stalker HT, Schmitz RJ, Scheffler BE, Leal-Bertioli SC, Xun X, Jackson SA, Michelmore R, Ozias-Akins P. The genome sequences of Arachis duranensis and Arachis ipaensis, the diploid ancestors of cultivated peanut. Nat Genet. 2016;48(4):438-46.

2. Chen XP, Lu Q, Liu H, Zhang JN, Hong YB, Lan HF, Li HF, Wang JP, Liu HY, Li SX, Pandey MK, Zhang ZK, Zhou GY, Yu JG, Zhang GQ, Yuan JQ, Li XY, Wen SJ, Meng FB, Yu SL, Wang XY, Siddiquw KHM, Liu ZJ, Paterson AH, Varshney RK, Liang XQ. Sequencing of cultivated peanut, Arachis hypogaea, yield insights into genome evolution and oil improvement. Mol Plant. 2019;12(7): 920-34.

3. Zhuang WJ, Chen $\mathrm{H}$, Yang $\mathrm{M}$, et al. The genome of cultivated peanut provides insight into legume karyotypes, polyploid evolution and crop domestication. Nat Genet. 2019;51:865-76.

4. Bertioli DJ, Jenkins J, Clevenger J, Dudchenko O, Gao D, Seijo G, SCM L-B, Ren L, Farmer AD, Pandey MK, Samoluk SS, Abernathy B, Agarwal G, Ballén- 
Taborda C, Cameron C, Campbell J, Chavarro C, Chitikineni A, Chu Y, Dash S, MEl B, Guo B, Huang W, Kim KD, Korani W, Lanciano S, Lui CG, Mirouze M, Moretzsohn MC, Pham M, Shin JH, Shirasawa K, Sinharoy S, Sreedasyam A, Weeks NT, Zhang X, Zheng Z, Sun Z, Froenicke L, Aiden EL, Michelmore R, Varshney RK, Holbrook CC, EKS C, Scheffler BE, Grimwood J, Ozias-Akins P, Cannon SB, Jackson SA, Schmutz J. The genome sequence of segmental allotetraploid peanut Arachis hypogaea. Nat Genet. 2019;51(5):877-84.

5. Alcorn JL, Punithalingam E, McCarthy GJP. Peanut net blotch caused by Didymosphaeria arachidicola (Chochryakov) comb. Nov Trans Br Mycol Soc. 1976;66:351-5.

6. Pettit RE, Philley GL, Smith DH. Peanut web blotch: II symptoms and host rang of pathogen. Peanut Science. 1986;13(1):27-30.

7. Marasas WFO, Pauer GD, Boerema GH. A serious leaf blotch disease of groundnuts (Arachis hypogaea L.) in southern Africa caused by Phoma arachidicola Sp. Nov Phytophylactica. 1974;6:195-202.

8. Taber RA, Petti RE, Philley GL. Peanut web blotch: I. cultural characteristics and identity of causal fungus. Peanut Science. 1984;11(2):109-14.

9. Pettit RE, Taber RA, Harrison AL. Ascochyta web-blotch of peanuts. Phytopathology. 1973;63:447.

10. Xu MX, Shi YM. Introduction the fungus of peanut web blotch in China. Peanut Sci Technol. 1990;1:19-20 (in Chinese).

11. Xu MX, Shi YM, Xu XJ. Cultural characteristics and identity of causal fungus of peanut web blotch. Acta Phytopathologica Sinica. 1992;22:270 (in (hinese).

12. Li JY, Li YZ, Jiao F, Wang YC. Studies the fungus of peanut web blotch in Shanxi Province. Peanut Sci Technol. 1991;1:1-6 (in Chinese).

13. Wang ZY, Wang SZ, Li HL, Yuan HX. Studies the fungus of peanut web blotch in Henan Province. J Henan Agri Sci. 1993;7:23-5 (in Chinese).

14. Blamey FPC, Chapman J, Young BW. Epiphytology of Phoma web blotch and Cercospora leaf-spot in Spanish groundnuts. Phytophylactica. 1977;9:63-4.

15. Mikunthan G. First report of web blotch of peanut caused by Phoma arachidicola in the dry zone of Sri Lanka. Plant Dis. 2007;81:832.

16. Zhang X, Xu ML, Wu JX, Dong WB, Chen DX, Wang L, Chi YC. Draft genome sequence of Phoma arachidicola Wb2 causing peanut web blotch in China. Curr Microbiol. 2019;76:200-6.

17. Smith $O D$, Smith DH, Simpson CE. Web blotch resistance in Arachis hypogaea L. Peanut Sci. 1979;6(2):99-101.

18. Zhang XY. Inheritance of main traits related to yield, quality and disease resistance and their QTLs mapping in peanut (Arachis hypogaea L.). Hangzhou: Zhejiang University; 2011. (Ph.D. Dissertation, in Chinese).

19. Novakazi F, Afanasenko O, Anisimova A, Platz GJ, Snowdon R, Kovaleva O, Zubkovich A, Ordon F. Genetic analysis of a worldwide barley collection for resistance to net form of net blotch disease (Pyrenophora teres $\mathrm{f}$. teres). Theor Appl Genet. 2019;132:2633-50.

20. Adhikari A, Steffenson BJ, Smith MJ, Dill-Macky R. Genomewide association mapping of seedling net form net blotch resistance in an Ethiopian and Eritrean barley collection. Crop Sci. 2019;59:1625-38.

21. Amezrou R, Verma RPS, Chao S, Brueggeman RS, Belqadi L, Arbaoui M, Rehman S, Gyawali S. Genome-wide association studies of net form of net blotch resistance at seedling and adult plant stages in spring barley collection. Mol Breed. 2018;38:1-14.

22. Richards JK, Friesen TL, Brueggeman RS. Association mapping utilizing diverse barley lines reveals net form net blotch seedling resistance/ susceptibility loci. Theor Appl Genet. 2017;130:915-27.

23. Wonneberger R, Ficke A, Lillemo M. Identification of quantitative trait loci associated with resistance to net form net blotch in a collection of Nordic barley germplasm. Theor Appl Genet. 2017;130:2025-43.

24. Adhikari A, Steffenson BJ, Smith KP, Smith M, Dill-Macky R. Identification of quantitative trait loci for net form net blotch resistance in contemporary barley breeding germplasm from the USA using genome-wide association mapping. Theor Appl Genet. 2020;133:1019-37.

25. Wang L, Zhou X, Ren X, Huang L, Luo H, Chen Y, Chen W, Liu N, Liao B, Lei $Y$, Yan L, Shen J, Jiang H. A major and stable QTL for bacterial wilt resistance on chromosome B02 identified using a high-density SNP-based genetic linkage map in cultivated Peanut Yuanza 9102 derived population. Front Genet. 2018;9:652.

26. Han S, Yuan M, Clevenger JP, Li C, Hagan A, Zhang X, Chen C, He G. A SNPBased Linkage Map Revealed QTLs for Resistance to Early and Late Leaf Spot Diseases in Peanut (Arachis hypogaea L.). Front Plant Sci. 2018;9:1012.
27. Wang Z, Huai D, Zhang Z, Cheng K, Kang Y, Wan L, Yan L, Jiang H, Lei Y, Liao B. Development of a high-density genetic map based on specific length amplified fragment sequencing and its application in quantitative trait loci analysis for yield-related traits in cultivated Peanut. Front Plant Sci. 2018;9:827.

28. Li HH, Zhang LY, Wang JK. Analysis and Answers to Frequently Asked Questions in Quantitative Trait Locus Mapping. ACTA. 2010;36(6):918-31.

29. Van Ooijen JW. JoinMap ${ }^{\otimes}$, Software for the calculation of genetic linkage maps in experimental populations, Kyazma BV. Wageningen, https:/www. kyazma. nl/index. php/JoinMap/; 2006.

30. Van Ooijen JW. MapQTL ${ }^{\bullet}$ 6, Software for the mapping of quantitative trait loci in experimental populations of diploid species. Wageningen, https:// www.kyazma.nl/index.php/MapQTL/: Kyazma BV; 2009.

31. Dash S, Cannon EKS, Kalberer SR, Farmer AD, Cannon SB. PeanutBase and other Bioinformatic resources for Peanut (chapter 8). In: Stalker HT, Wilson $R F$, editors. In peanuts genetics, processing, and utilization. San Diego: Elsevier Inc.; 2016. p. 241-52.

32. Tarr DEK, Alexander HM. TIR-NBS-LRR genes are rare in monocots: evidence from diverse monocot orders. BMC Res Notes. 2009:2:197.

33. Chandra S, Kazmi AZ, Ahmed Z, Roychowdhury G, Kumari V, Kumar M, Mukhopadhyay K. Genome-wide identification and characterization of NBARC resistant genes in wheat (Triticum aestivum L.) and their expression during leaf rust infection. Plant Cell Rep. 2017;36(7):1097-112.

34. Jones JD, Dangl JL. The plant immune system. Nature. 2006;444(7117):323-9.

35. Song H, Wang P, Li C, Han S, Zhao C, Xia H, Bi Y, Guo B, Zhang X, Wang X. Comparative analysis of NBS-LRR genes and their response to Aspergillus flavus in Arachis. PLoS One. 2017;12(2):e0171181.

36. Song H, Guo ZL, Hu XH, Qian L, Miao FH, Zhang XJ, Chen J. Evolutionary balance between LRR domain loss and young NBS-LRR genes production governs disease resistance in Arachis hypogaea cv. Tifrunner BMC Genomics. 2019;20:844.

37. Zhang L. Study on physiological and Biochemical Resistance of Peanut (Arachis hypogaea L.) against Web Blotch. Zhengzhou: Zhengzhou university; 2019. (Master thesis, in Chinese).

38. Ma KW. A new peanut variety Yuhua 4 with early maturity and high yield. J Henan Agric Sci. 1992;2:24 (in Chinese).

39. Yuan HX, Sun BJ, Li HL, Xing XP, Tang FS, Zhang XY. Identification of resistance to leaf spot in peanut cultivars (lines). J Henan Agric Sci. 2004;12: 35-8 (in Chinese with English abstract).

40. Wang ZY, Li SJ, Zhang XY, Gao M, Cui XW, Wang N, Sang SL. A method for identification of resistance to peanut web blotch. Patent of China. 2017; ZL201510708861.X. (in Chinese).

41. Yu SL. Chinese Peanut genetics and breeding. Shanghai: Shanghai Science and Technology Press; 2011. (in Chinese).

42. Huang X, Feng Q, Qian Q, Zhao Q, Wang L, Wang A, Guan J, Fan D, Weng Q, Huang T, Dong G, Sang T, Han B. High-throughput genotyping by whole-genome resequencing. Genome Res. 2009;19(6):1068-76.

\section{Publisher's Note}

Springer Nature remains neutral with regard to jurisdictional claims in published maps and institutional affiliations.

Ready to submit your research? Choose BMC and benefit from:

- fast, convenient online submission

- thorough peer review by experienced researchers in your field

- rapid publication on acceptance

- support for research data, including large and complex data types

- gold Open Access which fosters wider collaboration and increased citations

- maximum visibility for your research: over $100 \mathrm{M}$ website views per year

At BMC, research is always in progress.

Learn more biomedcentral.com/submissions 